\title{
Sub-second multi-channel magnetic control of select neural circuits in behaving flies
}

\author{
Authors: \\ Charles Sebesta ${ }^{1}$, Daniel Torres ${ }^{1}$, Boshuo Wang ${ }^{3}$, Zhongxi Li ${ }^{4}$, Guillaume Duret ${ }^{2}$, Kaiyi Jiang ${ }^{1}$, \\ Zhen Xiao ${ }^{9}$, Qingbo Zhang ${ }^{1}$, Vicki Colvin ${ }^{9}$, Stefan M Goetz ${ }^{3,4,5}$, Angel V Peterchev ${ }^{3,4,5,6}$, \\ Herman Dierick $^{7,8}$, Gang Bao ${ }^{1}$, Jacob T. Robinson ${ }^{1,2,7}$ \\ 1 Department of Bioengineering, Rice University, Houston, Texas, USA \\ 2 Department of Electrical and Computer Engineering, Rice University, Houston, Texas, USA \\ 3 Department of Psychiatry \& Behavioral Sciences, School of Medicine, Duke University, Durham, North Carolina, \\ USA \\ 4 Department of Electrical and Computer Engineering, School of Engineering, Duke University, Durham, North \\ Carolina, USA \\ 5 Department of Neurosurgery, School of Medicine, Duke University, Durham, North Carolina, USA \\ 6 Department of Biomedical Engineering, School of Engineering, Duke University, Durham, North Carolina, USA \\ 7 Department of Neuroscience, Baylor College of Medicine, Houston, Texas, USA \\ 8 Department of Human and Molecular Genetics, Baylor College of Medicine, Houston, Texas, USA \\ 9 Department of Chemistry, Brown University, Providence, Rhode Island, USA
}

\section{Abstract:}

Precisely timed activation of genetically targeted cells is a powerful tool for studying neural circuits and controlling cell-based therapies. Magnetic control of cell activity or "magnetogenetics" using magnetic nanoparticle heating of temperature-sensitive ion channels enables remote, non-invasive activation of neurons for deep-tissue applications and studies of freely behaving animals. However, the in vivo response time of thermal magnetogenetics is currently tens of seconds, which prevents the precise temporal modulation of neural activity similar to light-based optogenetics. Moreover, magnetogenetics has not provided a means to selectively activate multiple channels to drive behavior. Here we demonstrate that by combining magnetic nanoparticles with a rate-sensitive thermoreceptor (TRPA1-A) it is possible to achieve sub-second behavioral responses in Drosophila melanogaster. Furthermore, by tuning the properties of magnetic nanoparticles to respond to different magnetic field strengths and frequencies, we can achieve fast, multi-channel stimulation, analogous to optogenetic stimulation with different wavelengths of light. These results bring magnetogenetics closer to the temporal resolution and multiplexed stimulation possible with optogenetics while maintaining the minimal invasiveness and deep-tissue stimulation only possible by magnetic control.

\section{Main:}

Magnetic stimulation of genetically targeted cells, or "magnetogenetics", may enable researchers to apply a magnetic stimulus throughout the brain of a freely moving animal in a non-invasive manner to study circuits that are deep within the brain or distributed over large areas. The most reliable form of magnetogenetics relies on two components to be present in the tissue: synthetic magnetic nanoparticles that convert alternating magnetic fields into heat and thermoreceptors that convert the local heat into neural activity ${ }^{1-3}$. While there have been reports of magnetogenetic technologies that rely on purely genetically encoded proteins ${ }^{4-7}$, it is currently unclear how these magnetically sensitized chimeric proteins function ${ }^{8,9}$.

Compared to optical methods for stimulating genetically targeted cells (optogenetics), magnetogenetics offers unique advantages for deep volumetric targets. Most optical wavelengths are only effective at distances of a few $\mathrm{mm}$ from an optical source due to tissue scattering. In contrast, magnetic fields in the frequency range of $0.1-1 \mathrm{MHz}$ have very low attenuation in 
bone, air, and biological tissue ${ }^{10}$. This superior bone and tissue penetration of magnetic fields eliminates the need for invasive surgeries to introduce light probes typically required for optogenetic stimulation, interventions that can cause potential tissue damage from implantation and heat generation.

While magnetogenetics offers advantages including deep tissue volumetric stimulation and minimal invasiveness, the reported in vivo response time of magnetogenetic technologies is on the order of ten seconds - more than 1000-fold slower than optogenetic stimulation largely due to the thermoreceptors used. Previous experiments with membrane targeted cobalt-doped nanoparticles have shown latencies of $2.18 \pm 0.17 \mathrm{~s}$ in $\operatorname{trp} V 1^{+}$neurons in vitro and a $22.8 \pm 2.6 \mathrm{~s}$ latency in vivo via motor cortex stimulation resulting in an ambulatory response in $\operatorname{trpVI}^{+}$mice $^{2}$. Earlier experiments with undoped iron oxide nanoparticles showed a $\sim 5 \mathrm{~s}$ latency in vitro with $\operatorname{trp} \mathrm{VI}^{+}$neurons with upregulation of c-Fos expression in vivo on the order of minutes ${ }^{3}$. Existing magnetogenetic methods rely on thermoreceptors (e.g. TRPV1) that respond at temperatures several degrees above body temperature but heating the surrounding tissue to the threshold response temperature can take several seconds. These multi-second latencies prevent precise timing with behavioral or environmental cues that are essential for studying the relationship between neural activity and behavior. Magnetic activation of mechanoreceptors in contact with magnetic particles that move in response to a magnetic field offers a path to faster stimulation ${ }^{11}$, but the in vivo response time remains on the order of several seconds and requires micron-sized particles or aggregates that can be difficult to deliver in vivo ${ }^{12}$.

In this work we replaced the threshold thermoreceptors such as TRPV1 with a ratesensitive thermoreceptor to achieve fast response times similar to that which can be achieved with optogenetics. Since magnetic nanoparticle heating can increase the tissue temperature rapidly, it eliminates the wait-time required for the tissue to reach a threshold activation temperature when using thermoreceptors like TRPV1. Recent work demonstrates that Drosophila TRPA1-A activation is susceptible to the rate of temperature change and that rapid heating can lower the response threshold from $\sim 34.5^{\circ} \mathrm{C}$ to $\sim 29.1^{\circ} \mathrm{C}^{13}$. Additional experiments suggest that TRPA1-A is responsible for behavioral sensitivity to temperature changes of 0.01 ${ }^{\circ} \mathrm{C}$ in Drosophila ${ }^{3} / 18 / 2021$ 10:40:00 AM, $0.005{ }^{\circ} \mathrm{C}$ in C. elegans ${ }^{14}$, and $\sim 0.003{ }^{\circ} \mathrm{C}$ in snakes ${ }^{15}$, making it an ideal target receptor to confer rapid, sensitive thermosensation. Therefore, we selected TRPA1-A as the thermoreceptor to optimize magnetothermal channel activation and demonstrated sub-second multi-channel magnetogenetics in Drosophila. Since TRPA1-A is native, rate sensitive, and commonly used for thermogenetics with genetic lines readily available, our approach can be applied to a wide range of magnetogenetics studies of brain function.

To test if the Drosophila TRPA1-A rate-sensitive thermoreceptor would indeed enable a fast magnetogenetic activation we developed a system to measure Drosophila behavior under the influence of an alternating magnetic field (AMF). We generated fly strains that express thermal rate sensitive TRPA1-A channels in the fruitless circuit, which is known to control courtship behavior in males and can be easily observed by a lateral wing extension behavioral response, as was previously shown with optogenetic ${ }^{16}$ and thermogenetic ${ }^{17}$ techniques. Moreover, the behavior can be automatically tracked using pose estimation tools like DeepLabCut ${ }^{18}$ or FlyTracker ${ }^{19}$ eliminating observer bias. Instead of externally heating flies to activate the thermosensitive channel, we injected nanoparticles suspended into artificial Drosophila hemolymph (Fig. 1A, S1, see Methods). We placed the injected flies over an induction coil (Fig. 1B) and monitored the wing-opening behavior during AMF stimulation. The dissipated heat generated by the stimulated nanoparticles activates the dTRPA1-A protein channel (Fig. 1C, D). 
When we injected flies with $10 \mu \mathrm{g} / \mathrm{mL}$ of $15 \mathrm{~nm}$ cobalt-doped iron oxide nanoparticles and applied an AMF we observed a rapid increase in the wing angle with a response latency of $510 \pm 186 \mathrm{~ms}$ - more than 10 times faster than previous in vivo magnetogenetic latencies ${ }^{2,3}$ (Fig. 1E). To confirm that the response was driven by magnetic heating of the nanoparticles, we compared wing openings in flies injected with $19 \mathrm{~nm}$ magnetite particles to flies injected with 19 $\mathrm{nm}$ wüstite particles. These wüstite particles contain the same amount of iron as the magnetite particles, but lack the open hysteresis loop, and thus do not heat in an alternating magnetic field $^{20}$. When we applied an alternating magnetic field to these flies, we observed a sub-second wing opening response in the magnetite-injected flies, but no response in the wüstite injected flies confirming that the behavioral response we observe is mediated by magnetothermal heating (Fig. S2).

By comparing the fly response to fast and slow heating rates we were able to confirm that the fast response is indeed the result of the rate-sensitive properties of TRPA $1^{13}$. To perform experiments with different heating rates we first characterized the specific absorption rate (SAR) of $15 \mathrm{~nm}$ cobalt doped nanoparticles using an AMF at $49.9 \mathrm{kHz}$ at two different strengths (80 mT and $19 \mathrm{mT}$ ). These different AMF conditions result in a $\sim 10 \times$ difference in heating rate (Fig. 2A, B), which are calculated as averages over the AMF duration due to visible lag from thermal resistance in the IR probe. To assess the rate sensitivity of the behaving adults flies, we exposed the same set of flies to different temperature ramps by altering the magnetic field strength for different durations of time $\left(1.8 \mathrm{~s}\left(\sim 1.7^{\circ} \mathrm{C} / \mathrm{s}\right)\right.$ and $\left.20 \mathrm{~s}\left(\sim 0.17^{\circ} \mathrm{C} / \mathrm{s}\right)\right)$ while monitoring wing angle responses of adult males expressing TRPA1-A (Fig. 2C, D). These two heating conditions generate approximately the same amount of total heat to the animal, but only rapidly delivered heat results in reliable wing openings (Fig. 2E, F).

To generalize our findings, we also expressed the TRPA1 channel in a different neural circuit, producing a different behavioral effect. Driving expression of $d T R P A 1-A$ with $H b 9$ $G A L 4$ reliably induced a side-walking phenotype ${ }^{16}$ in the same behavior chamber under AMF control (Fig. 3).

We observed a clear, robust, and reversible side-walking phenotype during AMF stimulation among magnetite injected flies expressing dTRPAl-A in the Hb9-GALA circuit. In contrast, flies injecting with magnetically inert wüstite particles showed no response, demonstrating that the behavioral responses are due to specific nanoparticle heating and not an artifact of the magnetic field generation. The side walking behavior was more difficult to quantify than wing extension and took longer to develop. This increased latency may be due to the need to activate the peripheral nervous system where we expect fewer nanoparticles. Nevertheless, the magnetogenetic driven behavior was easily identified from the side-to-side behavior tracks (Fig. 3A) and the animal behavior videos (Video S3). FlyTracker generated a set of metrics for each track such as wing angle, velocity, angular velocity, and distance from the chamber wall. The combination of these FlyTracker metrics was used to train a machine learning model for side-walking behavior from videos for which side-walking behavior was hand annotated during thermal activation $\left(35^{\circ} \mathrm{C}\right)$ of flies expressing dTRPAl-A in the Hb9-GALA circuit using Janelia Automated Animal Behavior Annotator (JAABA) ${ }^{21}$. This model was then used to predict and annotate magnetically stimulated flies for similar behavior, providing a prediction score. Using FlyTracker and JAABA, we developed a classifier (see methods) to quantify the side-walking behavior before, during, and after stimulation. Quantification shows distinct and reversible modulation of side-walking behavior with magnetite injected flies but not wüstite injected control flies (Fig. 3B). 
We next explored whether magnetogenetic stimulation based on rate-sensitive thermoreceptors is compatible with reliable multichannel stimulation. By using nanoparticles that heat at different rates depending on the magnetic field conditions, we hypothesized that we could selectively activate flies injected with one type of nanoparticle (channel $1-$ Ch1) without stimulating flies injected with another type of nanoparticle (channel $2-\mathrm{Ch} 2$ ), and vice versa. This is analogous to optogenetic stimulation of different circuits using different wavelengths of light, but here the selectivity is determined by differences in the specific absorption rate (SAR) of nanoparticles that we design and synthesize. This multiplexing concept is supported by the recent finding that modulating amplitude and frequency of an alternating magnetic field can selectively heat nanoparticles with varying coercivity resulting in multiplexed magnetothermal heating in vitro ${ }^{22}$.

To create the first channel for magnetogenetic heating we developed a highly coercive nanoparticle by doping iron oxide with cobalt $\left(\mathrm{Fe}_{2.35} \mathrm{Co}_{0.65} \mathrm{O}_{4}\right)(\mathbf{F i g}$. 4A) which is able to generate a large amount of heat at a low frequency AMF with a high field strength (Ch1: $80 \mathrm{mT}$; 49.9 $\mathrm{kHz}$ ). To create the second channel, we used a recently developed iron oxide nanoclusters ${ }^{23}$ (Fig. 4B) with a low coercivity to generate a large amount of heat when exposed to a high frequency AMF with a low field strength $(\mathrm{Ch} 2: 12 \mathrm{mT} ; 555 \mathrm{kHz})$. These nanoparticles demonstrate a selectivity of $\sim 15 \times$ for cobalt nanoparticles in Ch1 (SAR $=829.37 \mathrm{~W} / \mathrm{g}$ for Cobalt; $50.57 \mathrm{~W} / \mathrm{g}$ for $\mathrm{Fe}_{3} \mathrm{O}_{4}$ clusters) and $\sim 10 \times$ for iron oxide nanoclusters in $\mathrm{Ch} 2$ (SAR $=31.60 \mathrm{~W} / \mathrm{g}$ for Cobalt; $302.30 \mathrm{~W} / \mathrm{g}$ for $\mathrm{Fe}_{3} \mathrm{O}_{4}$ clusters) when comparing heat generated over $3 \mathrm{~s}$ AMF stimulation (Fig. 4D, E) indicating 2 distinct channels available for magnetothermal heating. Crystal patterns for each particle confirmed by X-ray power diffraction (XRD) (Fig. S4).

When we measured wing angle in groups of flies injected with these different nanoparticles, we found that we could selectively drive wing extension in either group depending on which magnetic field stimulation channel we selected. Heating profiles of the nanoparticles were used to scale the injection concentration of each particle into fruit flies to achieve similar heating profiles in each magnetic field condition. We introduced a mixed group of flies injected with cobalt (orange circles) or iron oxide nanoclusters (blue circles) into the fly chamber and showed activation of behavior specifically in the optimized AMF and lack of a behavioral response in the off-target AMF condition (Fig. 5A-C). Exposure to 2 stimulations of Ch1 (2 s; 80 $\mathrm{mT} ; 49.9 \mathrm{kHz})$ followed by 2 stimulations of Ch2 $(2 \mathrm{~s} ; 12 \mathrm{mT} ; 555 \mathrm{kHz})$ show the selectivity on animal behavior response based on injected particle while maintaining sub-second latencies for each channel (Fig. 5B).

In summary, we report the first multiplexed magnetothermal activation of behavior in freely moving adult Drosophila melanogaster, and the first sub-second magnetogenetic response in vivo. This fast response was made possible by replacing the slow-response magnetothermal sensor TRPV1 by a rate-sensitive TRPA1 channel. Drosophila TRPA1-A is rate sensitive and native to flies. The magnetic activation of the channel drives behavior in vivo within $500 \mathrm{~ms}$ of stimulation for which we estimate thermal temperature increases in the tissue to be less than $1{ }^{\circ} \mathrm{C}$ based on nanoparticle heating and assuming a $10 \times$ dilution of nanoparticle concentration in fly hemolymph.

Future applications with targeted nanoparticles may enable multiplexing with similar channels with a heterogeneous population of target neurons or cells within the same volume. However, due to the size of the Drosophila nervous system, heat transfer limitations ${ }^{4,24}$, and the thermal rate required to activate these channels to direct behavior ${ }^{13}$, highly concentrated ferrofluids show the most promise for current neuronal stimulation applications. Further 
sensitization and optimization of the thermal rate response may make heterogeneous multichannel targeted activation of the nervous system possible by genetically targeting the nanoparticles to bind to specific membranes or channels.

The ideal magnetothermal sensor for mammalian stimulation at $37{ }^{\circ} \mathrm{C}$ may be found among the orthologous TRPA1 proteins in reptilian or avian species and/or through protein engineering, including site-specific mutagenesis or protein chimerization. This new magnetogenetic method depends on the Drosophila dTRPA1-A which is constitutively active at $37^{\circ} \mathrm{C}$. In order to adapt this approach to stimulate mammalian neurons, other channels with similar temperature rate sensitivities but higher threshold must be characterized or engineered. The heat responses of reptilian and avian TRPA1 channels have been described showing a conserved heat response in animals like the western clawed frog, chicken, green anole, rat snake, and rattlesnake $\mathrm{e}^{25-27}$, which might be promising candidates. These thermal responses have further proven to be heavily reliant on the ankyrin repeat $\mathrm{N}$-terminus domain in both Drosophila and snakes $^{13,28}$, which therefore constitute an ideal target for future protein engineering.

With the fast response and multiplexing abilities of magnetogenetics shown here we believe that this technology has the potential to rival optogenetics in terms of temporal resolution and multiplexed stimulation while maintaining the advantages of remote activation over large volumes of cells that may lie in deep tissue such as brain tissue occluded by the skull.

\section{Methods:}

\section{Generation of Biocompatible Magnetothermal Transducer and Control Particles}

As magnetite has shown promising biocompatibility ${ }^{29}$, we synthesized superparamagnetic iron oxide nanoparticles (SPIONs) consisting of magnetite nanocrystals and coated with a layer formed of copolymers of phospholipids and poly(ethylene glycol) (DSPE-PEG2K). The nanoparticles were synthesized, coated and functionalized in three consecutive steps similar to previously published work ${ }^{30}$. First, $4 \mathrm{~nm}$ magnetite nanocrystals were synthesized by thermal decomposition of iron acetylacetonate in a mixture of oleic acid and benzyl ether. The magnetite nanocrystals were then grown to $19 \mathrm{~nm}$ diameter by controllable seed-mediated growth in a mixture of iron acetylacetonate, oleic acid, and benzyl ether. The size distribution of the magnetite nanocrystals was then quantified by transmission electron microscopy (TEM). The magnetic properties and the crystal structure of the nanocrystals was then quantified by a superconducting quantum interference device (SQUID) and power X-ray diffraction (XRD).

The synthesized nanocrystals were then coated with a layer of oleic acid and only dispersible in a nonpolar solvent. To generate water-dispersible nanoparticles, the nanocrystals were coated with a mixture of DSPE-PEG2K using a dual-solvent exchange method ${ }^{31}$. The hydrodynamic size of conjugated nanoparticles was subsequently examined by dynamic light scattering. The heating efficiency of SPIONs was then examined by a magnetic inductive heating within the AMF device using an IR thermal probe (Lumasense Luxtron 812 and STF-2M Probe).

The cobalt-doped nanocrystals were made by multiple seed-mediated growth reactions using $5 \mathrm{~nm}$ iron oxide cores. They were synthesized through thermal decomposition, using 2 mmol $\mathrm{CoCl}_{2}, 4 \mathrm{mmol} \mathrm{Fe}(\mathrm{acac})_{3}, 25 \mathrm{mmol}$ oleic acid and $60 \mathrm{ml}$ benzyl ether as a solvent. The reaction was heated to $120^{\circ} \mathrm{C}$ for 30 minutes under a constant argon flow, then to $200^{\circ} \mathrm{C}$ for 2 hours and finally to reflux at $300{ }^{\circ} \mathrm{C}$ for 30 minutes. The product was purified through several acetone washes. The nanoparticle sizes were determined by HC TEM. Nanoparticles were then coated with DSPE-PEG2K by mixing the nanoparticles with PEG and adding DMSO. The 
reaction was then evaporated and transferred to water by a drop-wise addition of water and removal of the remaining DMSO by was done by centrifugation and ultracentrifugation.

The $40 \mathrm{~nm}$ iron oxide nanocrystal clusters were synthesized through the hydrothermal reaction. $\mathrm{FeCl}_{3} \square 6 \mathrm{H}_{2} \mathrm{O}$ (540 mg) was dissolved in ethylene glycol (20 mL) under vigorous magnetic stirring. Then poly(acrylic acid) $(250 \mathrm{mg})$, urea $(1200 \mathrm{mg})$, and ultra-high purity deionized water $(1.0 \mathrm{~mL},<18 \mathrm{~m} \Omega)$ were added to the solution. The mixture was vigorously stirred for $30 \mathrm{~min}$, yielding a transparent and bright yellow solution. The mixture was then transferred to a Teflon-lined stainless-steel autoclave, tightly sealed and then heated at $195{ }^{\circ} \mathrm{C}$ for 6 hours with a temperature ramp rate of $20^{\circ} \mathrm{C} / \mathrm{min}$. After the reaction mixture was cooled to room temperature, the product was collected using a magnet. The clusters were washed 6 times using ethanol and water to remove the unreacted reactants and byproducts and then dispersed in DI water. The sizes of clusters and primary particles were determined using TEM. More than 500 clusters were measured to determine the cluster dimensions.

Synthesis of the control wüstite $(\mathrm{FeO})$ nanocrystals, which are poor magnetothermal transducers, was achieved similarly to the magnetite synthesis with minor alterations. Benzyl ether was substituted with oleylamine, the initial reaction was lengthened with a reduced temperature, and a vacuum process was added. The wüstite nanocrystals were then purified with ethanol, surface treated via heating in oleylamine, and dispersed in toluene before they were coated with DSPE-PEG2K.

The samples for TEM measurements, both HC TEM and TITAN TEM, were prepared by diluting the samples and placing them in carbon-film grids. XRD samples were prepared by drying the nanoparticles under an argon flow and then pulverizing the resulting powder. SQUID measurements were done with coated samples by fixing the nanoparticles with calcium hemisulfate and enclosing them within a capsule to prevent movement. Doping percentages were determined by ICP-MS and the samples compared to the corresponding standard curves of iron and cobalt. Specific absorption rate $(\mathrm{SAR})$ is calculated as:

$$
\mathrm{SAR}=\mathrm{C} \cdot(\Delta \mathrm{T} / \Delta t) \cdot \rho^{-1}
$$

Where $\mathrm{C}$ is specific heat capacity of the media $\left(\mathrm{C}=4180 \mathrm{~J} \cdot \mathrm{kg}^{-1} \cdot \mathrm{K}^{-1}\right)$, T is the total temperature change during stimulation averaged over 3 stimulations, $t$ is the AMF stimulation time and $\rho$ is sample density measured by total metal concentration. Iron oxide nanoclusters were recorded at $10.09 \mathrm{mg}_{\text {Metal }} / \mathrm{mL}$ and cobalt doped nanoparticles were recorded at $9.58 \mathrm{mg}_{\text {Metal }} / \mathrm{mL}$. Temperature was measured with an IR thermal probe (Lumasense Luxtron 812 and STF-2M Probe) which is unaffected by magnetic fields.

\section{Fly stocks and husbandry}

Parental drosophila strains were obtained from the Bloomington Drosophila Stock Center: Fru-GAL4 (BL66696), UAS-TrpA1-A (BL26263), Hb9-GAL4 (BL32555). All flies were reared on cornmeal, molasses, sugar, yeast, and agar food, on a $16 \mathrm{~h} \mathrm{light} / 8 \mathrm{~h}$ dark cycle, and at room temperature $\left(22.5+/-0.5^{\circ} \mathrm{C}\right)$.

\section{Nanoinjection of Nanoparticles into Drosophila Heads:}

Different GALA driver lines were crossed to UAS-TRPAl flies and offspring with both GALA and $U A S$ components and single component controls were collected for injection. Nanoparticles were injected into adult male heads similar to a previously described protocol ${ }^{32}$. Males that were 1 to $5 \mathrm{~d}$ old were immobilized on ice and dropped head-down with an aspirator into a cylindrical hole punched with a pasteur pipette tip into a $2 \%$ ice-cooled agarose gel 
approximately $5 \mathrm{~mm}$ thick. The flies were then aspirated through the gel until the top of their head was flush with the gel surface. Five flies were immobilized in a gel at a time and transferred to a thermoelectric temperature controller (TE Technology Inc., Traverse City Michigan). Using a Nanoject II (Drummond Scientific Company), and borosilicate needle pulled on a Model P-97 needle puller (Sutter Instruments), nanoparticles resuspended in artificial Drosophila hemolymph $^{32}$ were aspirated into the needle. Using a micromanipulator (Narishige, Model M152) attached to a fixed post to move the Nanoject in 3 dimensions, the needle tip was placed just above the top of the fly head sticking out of the refrigerated gel and positioned between the 3 ocelli at a $45^{\circ}$ angle. The flies were then injected by gently pushing the needle forward until it penetrated the cuticle between the ocelli. Approximately $200 \mathrm{~nL}$ of nanoparticles suspended in artificial hemolymph were injected directly into the brain and flies were aspirated through the gel into an empty vial containing standard fly food. Iron oxide nanoparticles of $19 \mathrm{~nm}$ diameter were injected at $10 \mathrm{mg} / \mathrm{mL}$, cobalt doped iron oxide particles were injected at $10 \mathrm{mg} / \mathrm{mL}$, and $40 \mathrm{~nm}$ iron oxide clusters were injected at $25 \mathrm{mg} / \mathrm{mL}$. Animals were then allowed to recover overnight before being placed in a behavior chamber and stimulated with AMF (Figure 1).

\section{AMF Stimulation of Drosophila}

Animals were given at least 16 hours to recover from nanoparticle injection before being loaded into the AMF generator. Experimental and control animals were each placed into one of five cylindrical arenas (12 $\mathrm{mm}$ diameter) in the behavioral chambers within a $50 \mathrm{~mm}$ diameter enclosure by aspiration through a small hole cut into an acrylic cover that can rotate over each arena. This 3D printed behavior chamber is then placed into a 3D printed chamber holder which places the animals in the center of a 17 turn $50 \mathrm{~mm}$ inner diameter (ID) coil (Nanotherics Magnetherm) for the courtship behavior using $19 \mathrm{~nm}$ magnetite (Figure S2) or a custom high powered 6 turn $50 \mathrm{~mm}$ ID coil (Fluxtrol/AMF Lifesystems) for sidewalking behavior experiments via inductive heating. Stimulation of cobalt nanoparticles and iron oxide nanoclusters were performed by placing the chamber $\sim 6 \mathrm{~mm}$ above the surface of a 6-turn 57.7 mm ID Hi-Flux coil with a ferrite core $(\mu=2300)$ (MSI Automation) driven by a custom FPGAcontrolled hybrid Silicon-Gallium-Nitride transistor based power electronics system (Duke University), which can generate AMF in the same coil at several distinct frequency channels spanning $50 \mathrm{kHz}$ to $\sim 5 \mathrm{MHz}$ and rapidly switching between the channels on millisecond time scale. The camera (Basler acA2000-165umNIR, 50mm F1.8 Edmund optics 86574) was then fixed above the animals and synced with the alternating magnetic field via TTL triggers to temporally align behavioral recordings with magnetic field generation. Frequency was set by the machine while field strength was measured by a magnetic field probe placed in the same location as the fly behavior chamber (Fluxtrol).

Thermal ramp demonstration used 2 stimulations of $1.8 \mathrm{~s}$ duration at $80 \mathrm{mT}$ and $49.9 \mathrm{kHz}$ for fast heating and $20 \mathrm{~s}$ duration at $19 \mathrm{mT}$ and $49.9 \mathrm{kHz}$ for slow heating. The inter stimulation intervals were 30 or $60 s$ for fast and slow heating respectively. Multichannel demonstration used exposure to 2 stimulations of $\mathrm{Ch} 1(2 \mathrm{~s} ; 80 \mathrm{mT} ; 49.9 \mathrm{kHz})$ followed by 2 stimulations of Ch2 (2 $\mathrm{s} ; 12 \mathrm{mT} ; 555 \mathrm{kHz}$ ) with inter stimulation interval of $10 \mathrm{~s}$. The video recording is paused for less than 1 second in the multiplexing recording $(\mathrm{t}=20 \mathrm{~s})$ to switch the stimulation protocol on the software from $\mathrm{Ch} 1$ to $\mathrm{Ch} 2$.

\section{Automated Analysis of Behavioral Phenotypes in Drosophila}


Animals were given at least 5 minutes to adjust to the behavior chamber before stimulation with pulsed cycles of alternating magnetic fields. Backlit videos of flies were analyzed using the Caltech FlyTracker ${ }^{19}$ to automatically identify the fly position, orientation, and wing/leg extensions. These data were then be analyzed on a frame-to-frame basis in MATLAB (MathWorks) for specific phenotypes (e.g. maximum wing angle for the wing extension phenotype and fly position for side-walking phenotype) or used with machine learning tools like Janelia Automated Animal Behavior Annotator (JAABA) to train complex behaviors that take place over a series of frames (lateral movement phenotype in side-walking) which enables linear regression models to predict the occurrence of the phenotype. Multiplexed animal behavior was analyzed using DeepLabCut ${ }^{18}$ for dynamic tracking of flies with visible shadows introduced by front lighting needed to illuminate flies above the ferrite core. The animal analysis was trained using a skeleton labeling the head, neck, tip of each wing, and abdomen. Wing angle was calculated between the neck and each wingtip.

\section{Acknowledgments}

This research was developed with funding from the Defense Advanced Research Projects Agency (DARPA) of the United States of America, Contract No. N66001-19-C-4020. The views, opinions and/or findings expressed are those of the authors and should not be interpreted as representing the official views or policies of the Department of Defense or the U.S.A. Government.

This work was funded in part by the National Science Foundation - NSF Neuronex innovation award 1707562, grant C-1963 from the Welch Foundation, and National Institutes of Health under award number RO1MH107474. Any opinions, findings, and conclusions or recommendations expressed in this material are those of the author(s) and do not necessarily reflect the views of the National Science Foundation or National Institutes of Health.

We also thank Junsang Moon and Polina Anikeeva (MIT) for useful discussions and guidance with magnetic multiplexing. 
1. Huang, H., Delikanli, S., Zeng, H., Ferkey, D. M. \& Pralle, A. Remote control of ion channels and neurons through magnetic-field heating of nanoparticles. Nat. Nanotechnol. 5, 602-6 (2010).

2. Munshi, R. et al. Magnetothermal genetic deep brain stimulation of motor behaviors in awake, freely moving mice. eLife (2017) doi:10.7554/eLife.27069.

3. Chen, R., Romero, G., Christiansen, M. G., Mohr, A. \& Anikeeva, P. Wireless magnetothermal deep brain stimulation. Science 347, 1477-1480 (2015).

4. Duret, G. et al. Magnetic Entropy as a Proposed Gating Mechanism for Magnetogenetic Ion Channels. Biophys. J. 116, 454-468 (2019).

5. Stanley, S. A. et al. Radio-wave heating of iron oxide nanoparticles can regulate plasma glucose in mice. Science (2012) doi:10.1126/science.1216753.

6. Stanley, S. A., Sauer, J., Kane, R. S., Dordick, J. S. \& Friedman, J. M. Remote regulation of glucose homeostasis in mice using genetically encoded nanoparticles. Nat. Med. 21, 92-98 (2015).

7. Wheeler, M. A. et al. Genetically targeted magnetic control of the nervous system. Nat. Neurosci. 19, 756-761 (2016).

8. Wang, G. et al. Revaluation of magnetic properties of Magneto, MagR and aGFP-TRPV1/GFP-ferritin. http://biorxiv.org/lookup/doi/10.1101/737254 (2019) doi:10.1101/737254.

9. Kole, K. et al. Assessing the utility of MAGNETO to control neuronal excitability in the somatosensory cortex. bioRxiv 762559 (2019) doi:10.1101/762559. 
10. Young, J. H. H., Wang, M.-T. T. \& Brezovich, I. A. A. Frequency/depth-penetration considerations in hyperthermia by magnetically induced currents. Electron. Lett. (1980) doi:10.1049/el:19800255.

11. Gregurec, D. et al. Magnetic Vortex Nanodiscs Enable Remote Magnetomechanical Neural Stimulation. ACS Nano 14, 8036-8045 (2020).

12. Lee, J. et al. Non-contact long-range magnetic stimulation of mechanosensitive ion channels in freely moving animals. Nat. Mater. 1-8 (2021) doi:10.1038/s41563-020-00896-y.

13. Luo, J., Shen, W. L. \& Montell, C. TRPA1 mediates sensation of the rate of temperature change in Drosophila larvae. Nat. Neurosci. 20, 34-41 (2017).

14. Rosenzweig, M. et al. The Drosophila ortholog of vertebrate TRPA1 regulates thermotaxis. Genes Dev. (2005) doi:10.1101/gad.1278205.

15. BULLOCK, T. H. \& DIECKE, F. P. Properties of an infra-red receptor. J. Physiol. (1956) doi:10.1113/jphysiol.1956.sp005624.

16. Inagaki, H. K. et al. Optogenetic control of Drosophila using a red-shifted channelrhodopsin reveals experience-dependent influences on courtship. Nat. Methods 11, 325-32 (2014).

17. von Philipsborn, A. C. et al. Neuronal Control of Drosophila Courtship Song. Neuron (2011) doi:10.1016/j.neuron.2011.01.011.

18. Mathis, A. et al. DeepLabCut: markerless pose estimation of user-defined body parts with deep learning. Nat. Neurosci. 21, 1281-1289 (2018).

19. Eyjolfsdottir, E. et al. Detecting social actions of fruit flies. in Lecture Notes in Computer Science (including subseries Lecture Notes in Artificial Intelligence and Lecture Notes in Bioinformatics) (2014). doi:10.1007/978-3-319-10605-2_50. 
20. Tong, S., Ren, B., Zheng, Z., Shen, H. \& Bao, G. Tiny grains give huge gains: Nanocrystalbased signal amplification for biomolecule detection. ACS Nano (2013) doi:10.1021/nn400733t.

21. Kabra, M., Robie, A. A., Rivera-Alba, M., Branson, S. \& Branson, K. JAABA: Interactive machine learning for automatic annotation of animal behavior. Nat. Methods (2013) doi:10.1038/nmeth.2281.

22. Moon, J. et al. Magnetothermal Multiplexing for Selective Remote Control of Cell Signaling. Adv. Funct. Mater. 30, 2000577 (2020).

23. Xiao, Z. et al. Libraries of Uniform Magnetic Multicore Nanoparticles with Tunable Dimensions for Biomedical and Photonic Applications. ACS Appl. Mater. Interfaces 12, 41932-41941 (2020).

24. Riedinger, A. et al. Subnanometer local temperature probing and remotely controlled drug release based on Azo-functionalized iron oxide nanoparticles. Nano Lett. (2013) doi:10.1021/n1400188q.

25. Saito, S. et al. Analysis of transient receptor potential ankyrin 1 (TRPA1) in frogs and lizards illuminates both nociceptive heat and chemical sensitivities and coexpression with TRP vanilloid 1 (TRPV1) in ancestral vertebrates. J. Biol. Chem. (2012) doi:10.1074/jbc.M112.362194.

26. Saito, S. et al. Heat and noxious chemical sensor, chicken TRPA1, as a target of bird repellents and identification of its structural determinants by multispecies functional comparison. Mol. Biol. Evol. (2014) doi:10.1093/molbev/msu001.

27. Gracheva, E. O. et al. Molecular basis of infrared detection by snakes. Nature (2010) doi:10.1038/nature08943. 
28. Geng, J., Liang, D., Jiang, K. \& Zhang, P. Molecular evolution of the infrared sensory gene TRPA1 in snakes and implications for functional studies. PLoS ONE (2011) doi:10.1371/journal.pone.0028644.

29. Hanini, A. et al. Evaluation of iron oxide nanoparticle biocompatibility. Int. J. Nanomedicine 6, 787-794 (2011).

30. Tong, S., Quinto, C. A., Zhang, L., Mohindra, P. \& Bao, G. Size-Dependent Heating of Magnetic Iron Oxide Nanoparticles. ACS Nano (2017) doi:10.1021/acsnano.7b01762.

31. Tong, S., Hou, S., Ren, B., Zheng, Z. \& Bao, G. Self-assembly of phospholipid-PEG coating on nanoparticles through dual solvent exchange. Nano Lett. (2011) doi:10.1021/nl201978c.

32. Lima, S. Q. \& Miesenböck, G. Remote control of behavior through genetically targeted photostimulation of neurons. Cell 121, 141-152 (2005). 


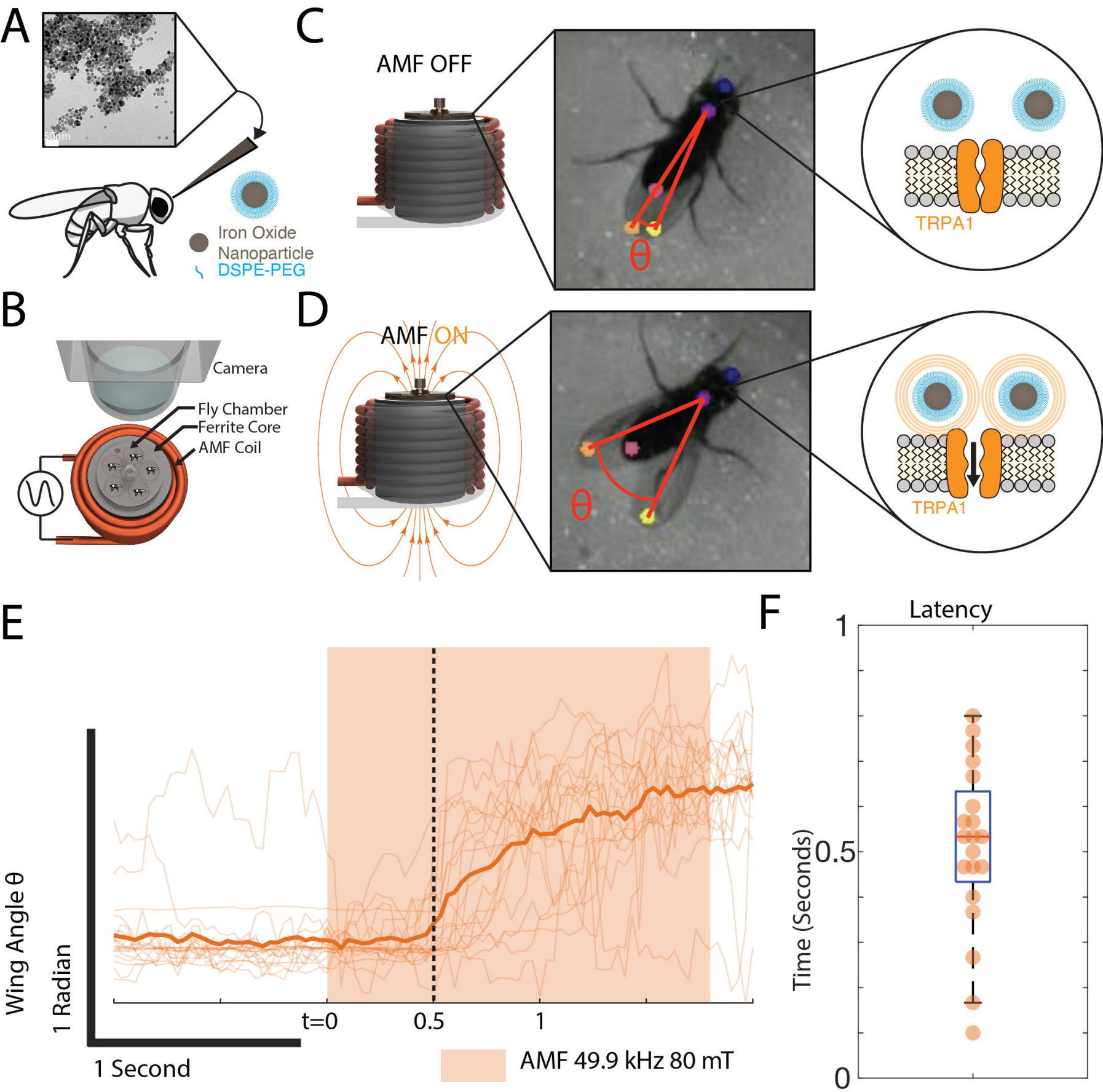


$\begin{array}{llll}\text { A } & 0.17^{\circ} \mathrm{C} / \mathrm{sec} & \text { B } & 1.7^{\circ} \mathrm{C} / \mathrm{sec}\end{array}$

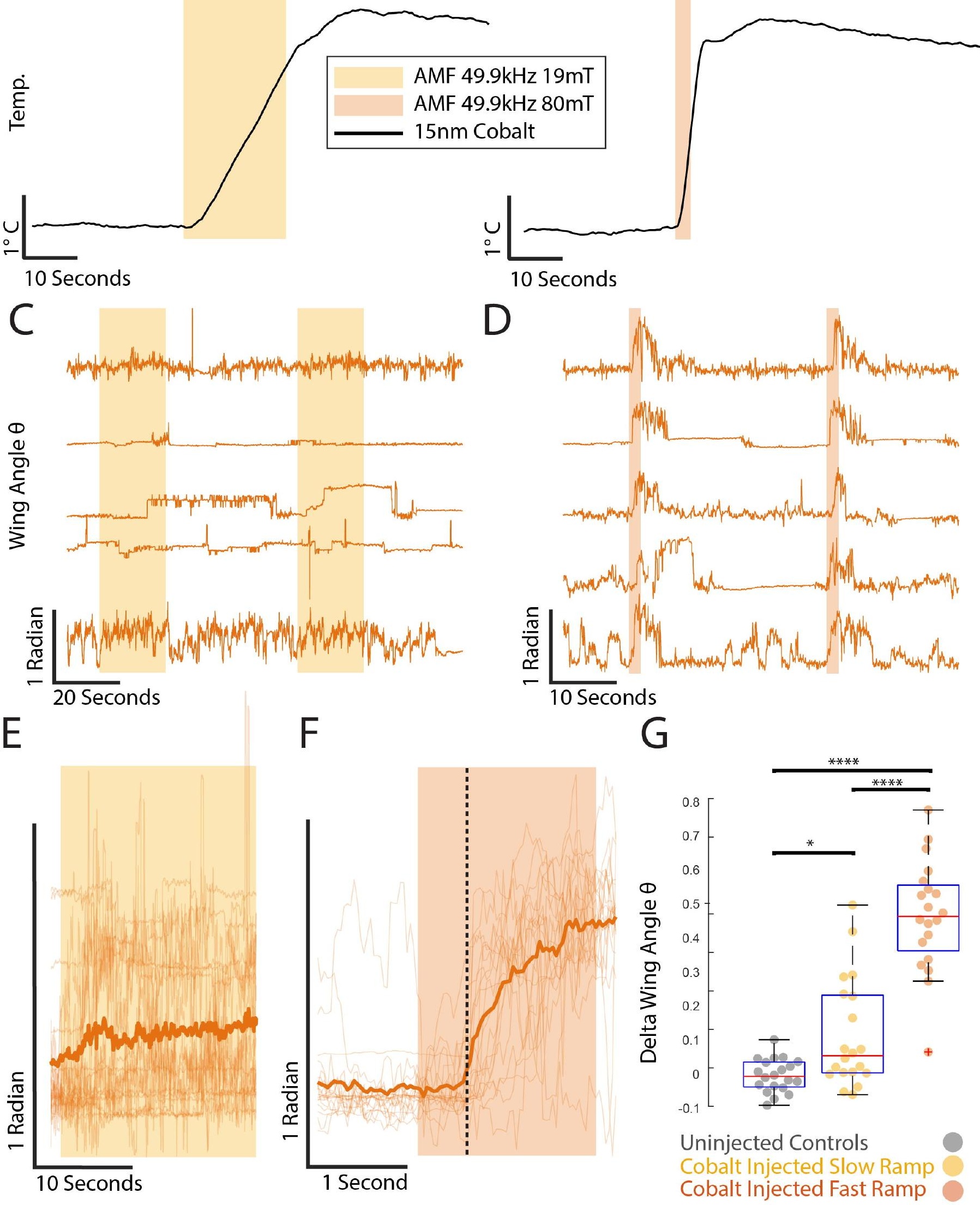



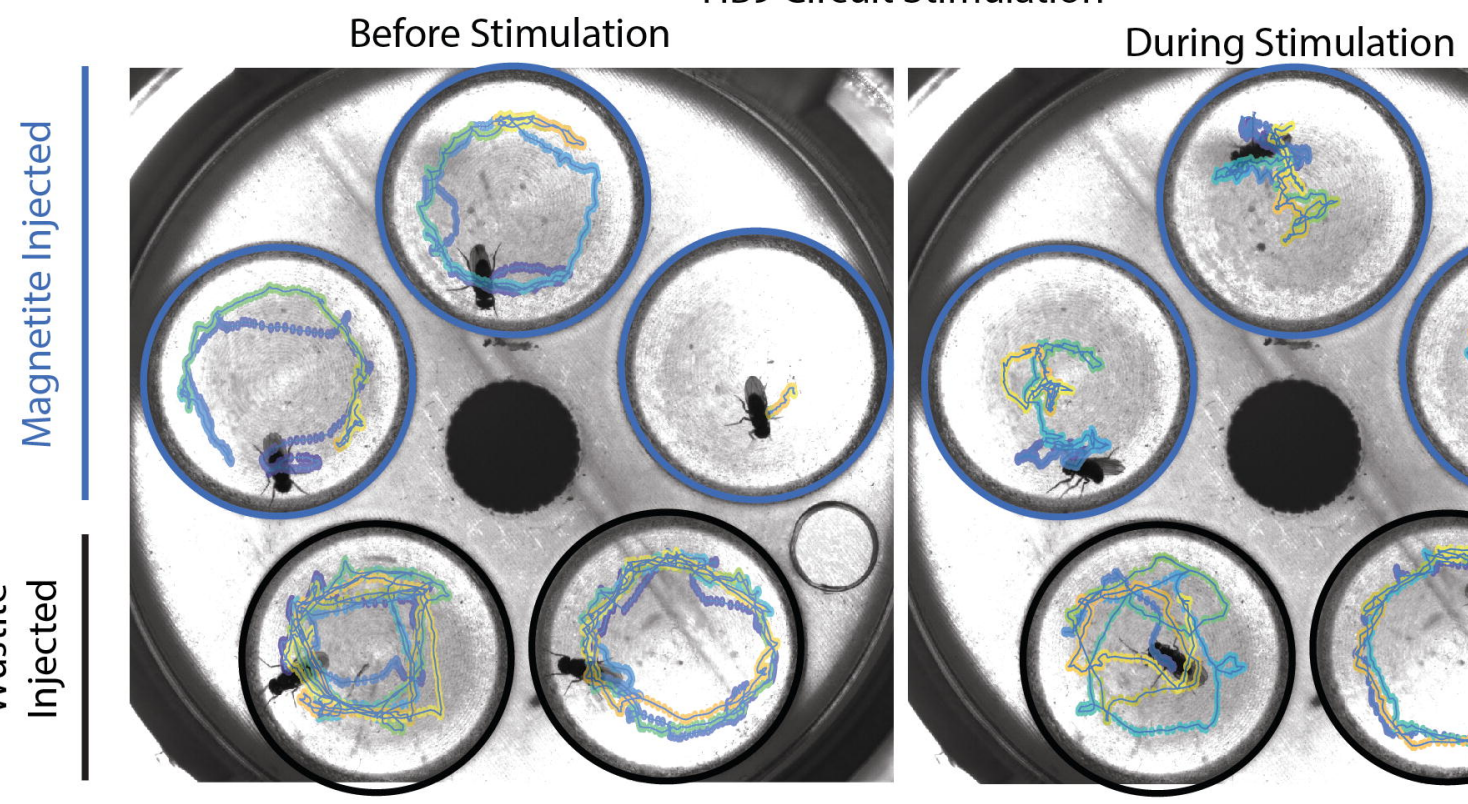

B

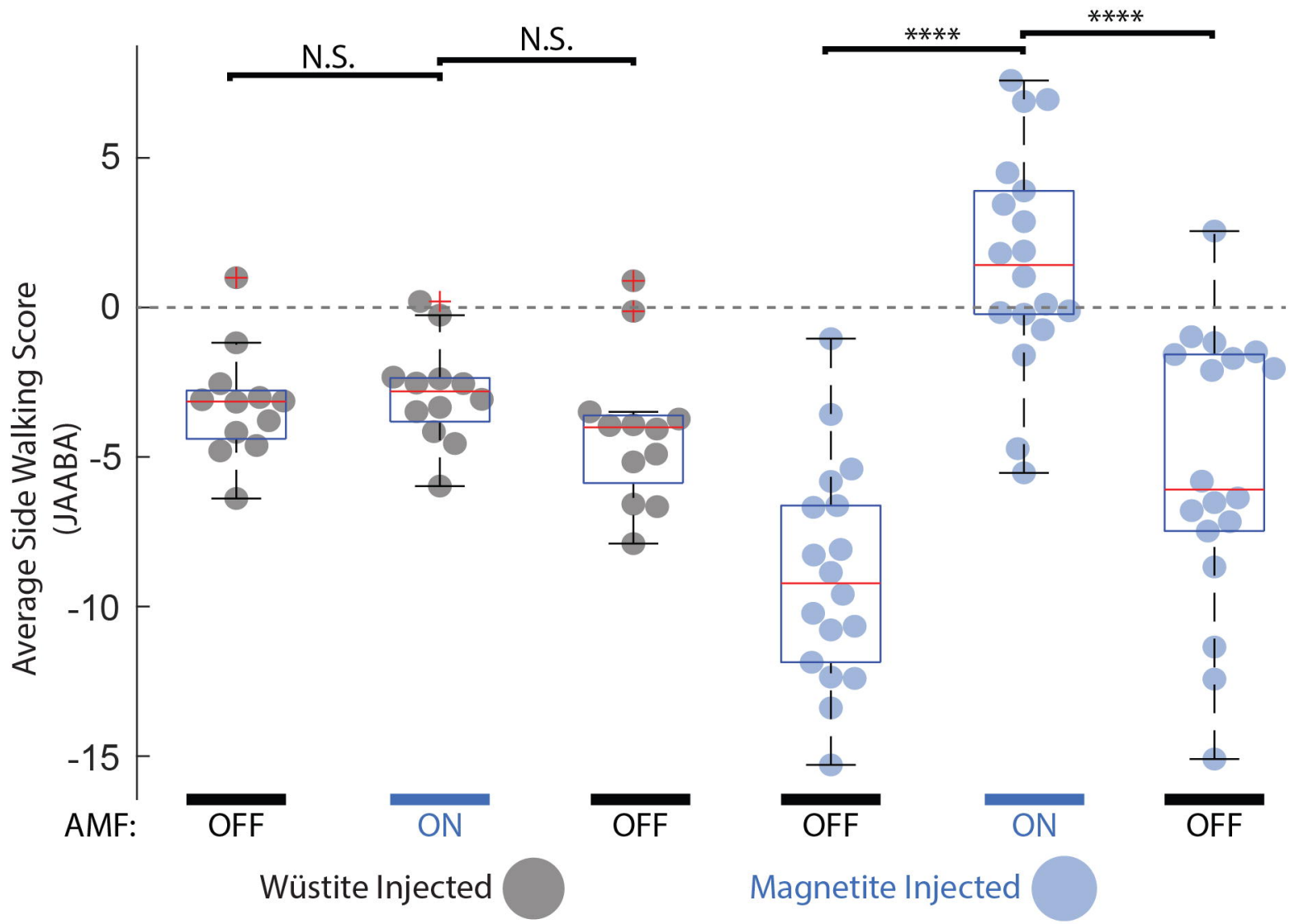


B Channel 2:

$15 \mathrm{~nm}$ Cobalt Nanoparticles

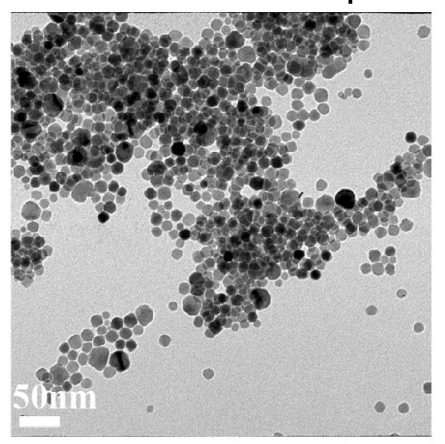

$40 \mathrm{~nm}$ Iron Oxide Clusters

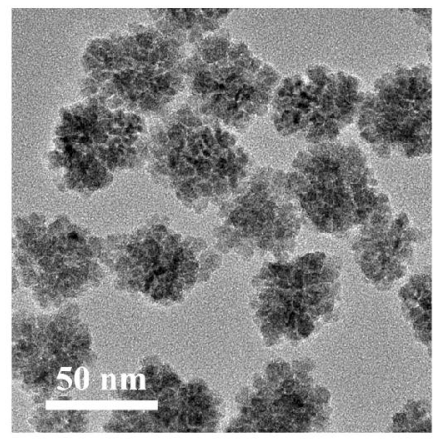

Magnetization Curve (5 K)

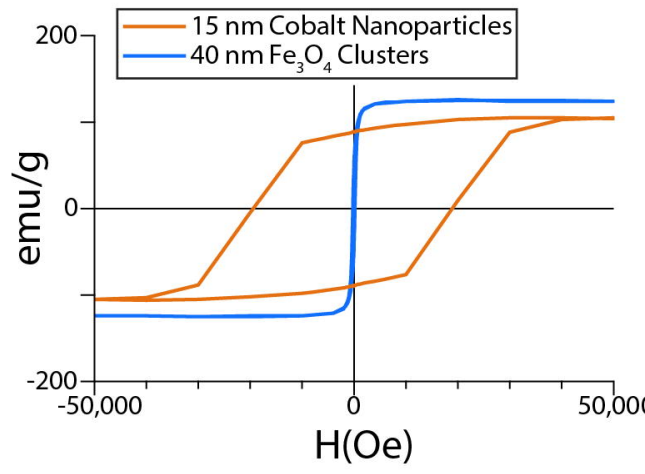

D

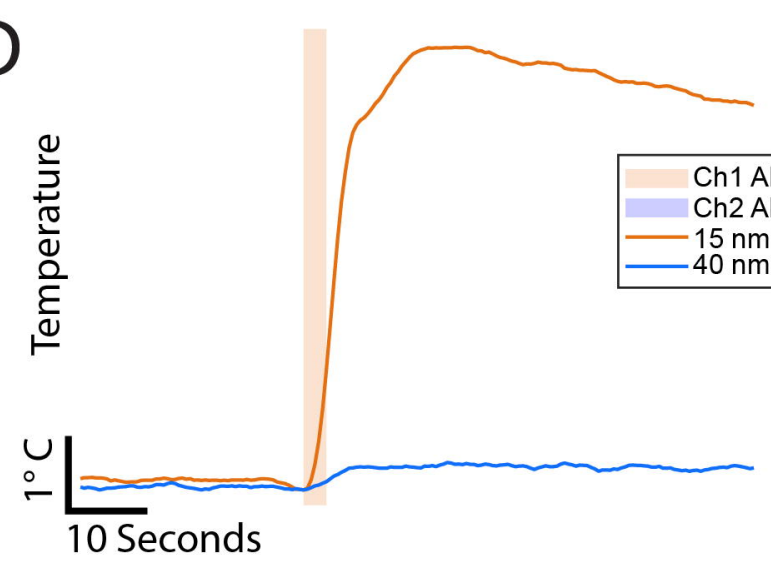

$E$

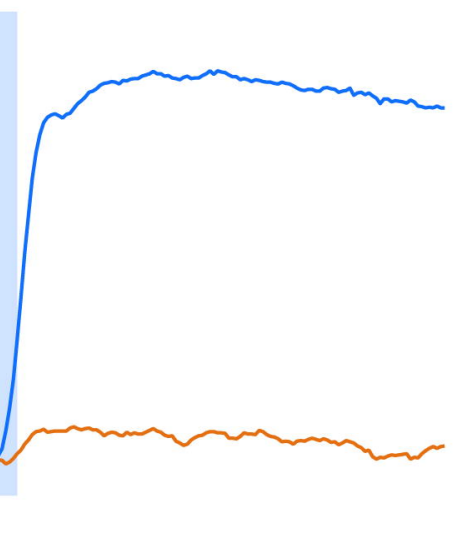



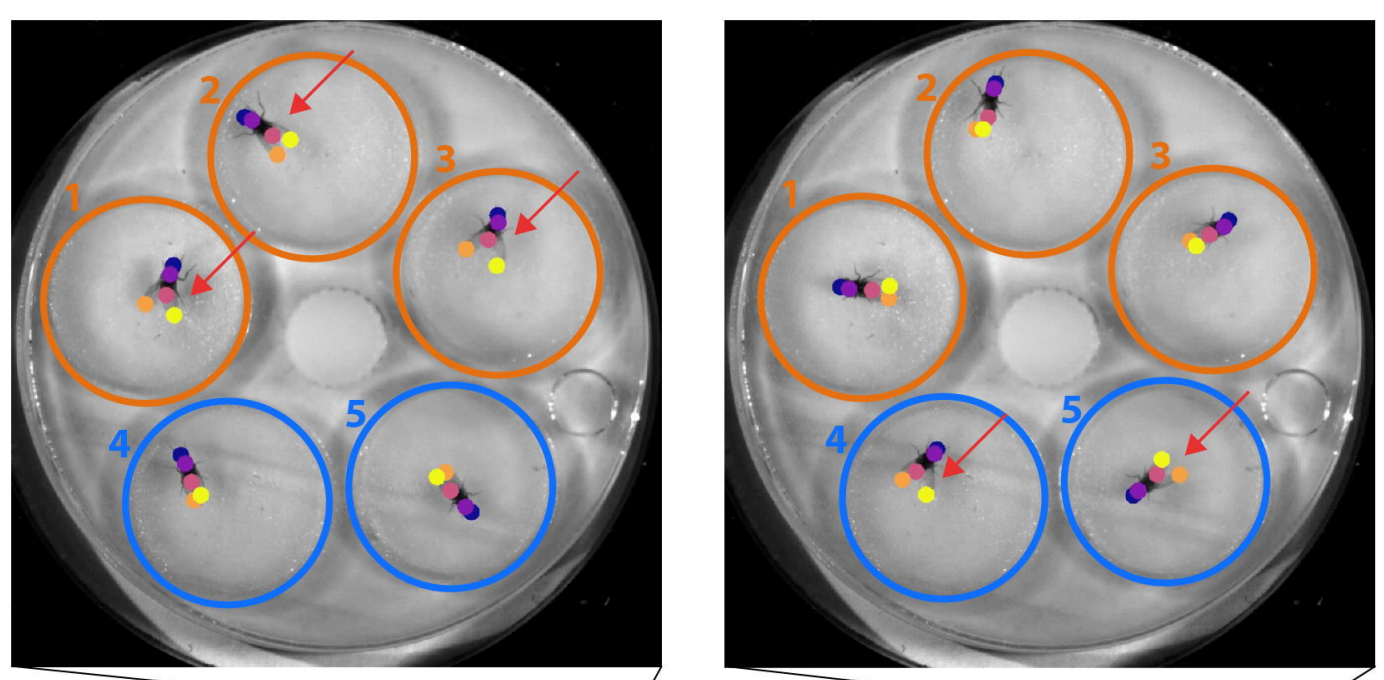

Е

흥

응 언

ह

ㄴ

B
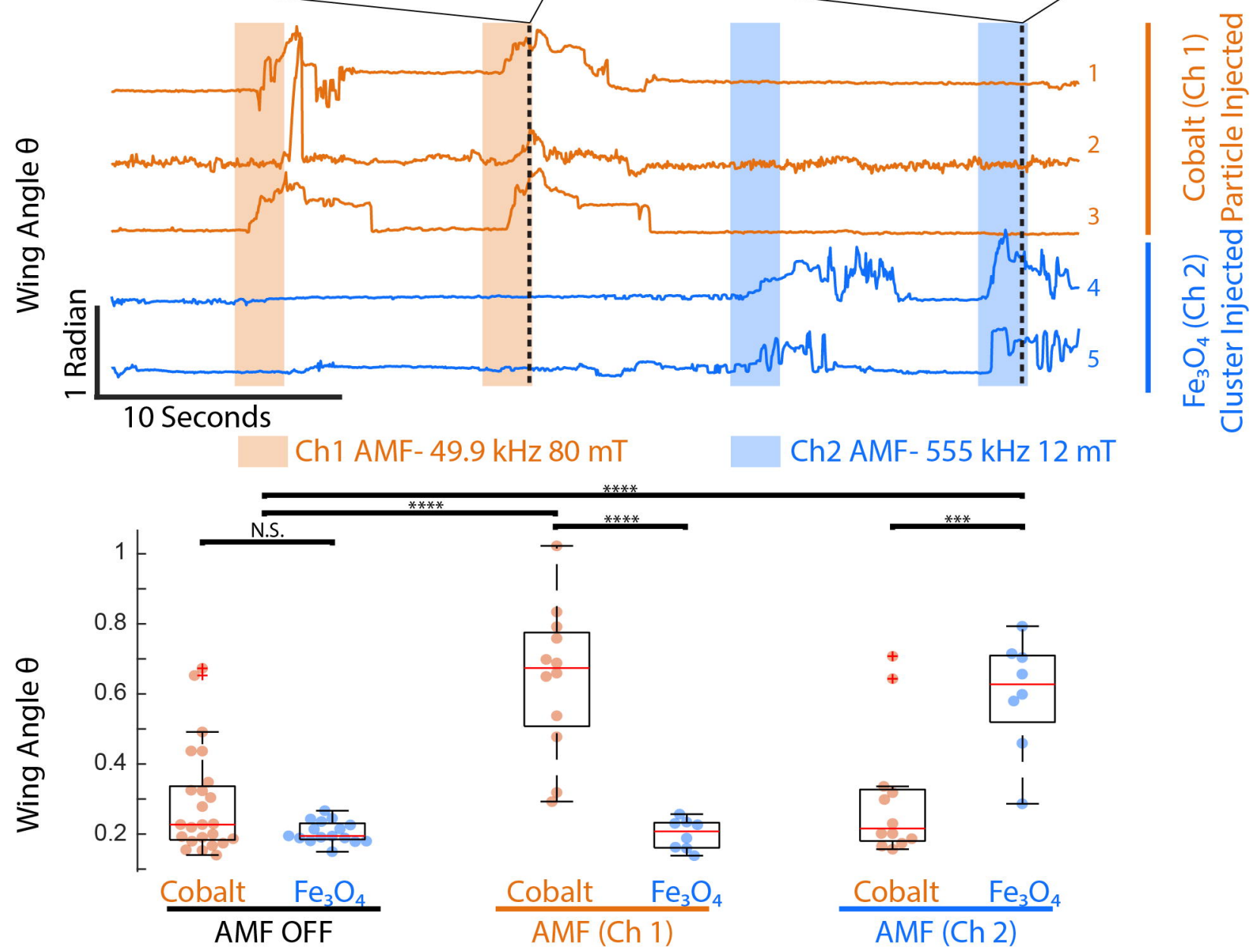